DVD BRIEFLY NOTED

\section{Dissection of the Human Brain}

L. Heimer. Sinauer Associates; 2007, running time: 2 hours 43 minutes, $\$ 75.00$.

This DVD, authored by Lennart Heimer, MD, from the University of Virginia, is a demonstration of blunt brain dissection. With a running time of 2 hours and 43 minutes, it is divided into 3 sections. The first section demonstrates the major white matter tracts; the second, surface anatomy and deeper gray matter of the brain; and the third, major pathways such as the corticospinal tracts, sensory pathways, visual system pathways, brain stem, basal ganglionic structures, and the limbic system, among others. In some areas, the dissections are accompanied by diagrams showing structures that are to be seen.

What makes this DVD valuable is its reinforcement of the real-life location of many important tracts, including those that subserve the motor, sensory, and emotional circuits. Although the DVD is educational in its own right, the addition of accompanying MR images, particularly those demonstrating diffusion tensor imaging or selected tractography, would have made it more germane to practicing neuroradiologists, neurologists, and neurosurgeons. Perhaps in an updated DVD, Dr. Heimer could involve his neuroradiology colleagues at the University of Virginia to secure these correlative images. It would then become a more compelling DVD.

DOI 10.3174/ajnr.A1551 